\section{CPh 04}

RELEVANCE OF STEROID RECEPTORS FOR PROGNOSIS AND THERAPY IN BREAST CANCER.

W. Eiermann

The concentration of receptors ( $E R$ and $P R$ ) in breast cancer is now well established as an index for predicting the subsequent response of the metastatic disease to endocrine therapy. Receptor concentration is influenced by menstrual status, tumor cellularity and grade and rises with increasing age. Adjuvant endocrine therapy should be made with the knowledge of ER and PR on the primary tumor. Though most studies showed no benefit for premenopausal women, in postmenopausal receptor pos. tumors and pos. axillary nodes numerous studies demonstrated a benefit of tamoxifen on disease free survival, obviously also depending on receptor quantity.

Several reports have suggested that early recurrence is associated with a lack of receptors in the primary tumor. Data from others have failed to confirm this. This conflicting data are mostly due to the ratrospective nature of the studies. By contrast, there is general agreement that patients whose tumor contains receptors survive longer after primary therapy than those who do not. Wether this beneficial effect is the result of a longer disease free interval or a prolongation of survival after relapse due to Iocation of metastatic disease, orboth, is not clear. Since receptor containing tumors are more likely to respond to endocrine therapy it is possible that the survival improvement following mastectomy reflects the response to treatment given for recurrent disease.

Frauenklinik, Klinikum Großhadern der LMU München, Marchioninistr. 15, 8000 Mïnchen 70

\section{CPh 05}

CLINICAL RELLVANCE OF ANDROGEN RECEPTOR DETERMENATION IN HUMAN BREAST CANCER.

K.- D. Schulz

The role of androgens in the growth control of human breast cancer is not yet quite clear. In the past, in vivo and in vitro observations showed the dose-dependent inhibition or stimlation of tumor proliferation by different androgenic compounds. The mode of action however, or the intracellular interactions with other hormones need further explanations and investigations. The existence of androgen binding proteins in nearly $50 \%$ of all tumors investigated has been reported in the meantime by different groups. Some data demonstrate the tendency, that androgen receptors possess possibly prognostic importance. However, the prediction of tumor response to hormonal or cytotoxic treatment seems to be impossible by the denonstration of androgen binding proteins in individual human breast cancers.

only the special prediction of tumor response to antiandrogens might be possible using androgen receptor assay.

Dept. Gynecol. Obstet., University of Marburg, Filgrimstein 3, D-3550 Marburg 1.

\section{$\mathrm{CPh} 06$}

THERAPEUTIC AND PROGNOSTIC IMPLICATIONS OF CORTICOSTEROID-RECEPTOR ASSAY IN LYMPHOID MALIGNANCIES A.D.Ho

Glucocorticoids (G) are part of most therapy regimens used for patients with lymphoid malignancies. The presence of specific receptors and the formation of glucocorticoid-receptor complexes seem to be required for hormone action in glucocorticoid sensitive tissues. Consequently, we have undertaken a series of studies on the utility of measuring glucocorticoid-receptors (GR) in predicting response to $G$ in leukemia or lymphoma. Leukemic cells from 24 patients with acute lymphatic leukemia (ALL) and 1 ymphoma cells or tissues from $15 \mathrm{pa}-$ tients with malignant lymphoma (NHL) were examined for glucocorticoid receptors. The patients were then treated with dexamethasone or prednisone as a single agent for 5 to 10 days. In patients with ALL 13 of the 16 patients ( $81 \%$ ) with high levels of GR (> 5000 binding sites/ce11) and none of the 8 patients with low levels of receptors ( $<5000$ binding sites/cell) responded to this initial therapy. Furthermore complete remissions upon multi-chemotherapy could be achieved in 12 of the 16 patients with high GR and 2 of the 8 patients with low GR. In patients with NHL, 7 of the 9 patients $(78 \%)$ with high GR levels and none of the 6 patients with low $G R$ levels responded to $G$ monotherapy. Thus our data suggest that study of GR in 1 ymphoid malignancies may allow selection of those patients who could benefit from glucocorticoid as part of combination chemotherapy. Knowledge of GR in these malignancies has also lead to the development of conjugates of steroid and cytostatic drugs. Prednimustine, a covalent conjugate of prednisolone and chlorambucil, has already been used in clinical trials for patients with Iymphoma and breast cancer. We are concurrently studying the correlation between GR and response to this drug in patients with low-grade malignant lymphoma.

Med.Univ.-Polikinik, Hospitalstraße 3, 6900 Heidelberg

\section{CPh 07}

PHARMACOCINATICAL AND CLINICAL INVESTIGATIONS ABOUT THE EFFICACY OF HD-MPA-THERAPY.

K. Possinger, L. Jaspers, L. Schmid, W. Wi 1 manns.

High-dose (HD) MPA treatment seems to induce higher objective remission rates in patients (pts.) with advanced breast cancer compared to administering traditional low-doses. The purpose of our study is to determine the response rate and remission duration of high-dose MPA therapy on the one hand, and to compare the therapy response with the plasma level (p.1.) profiles of MPA, Cortisol and Prolactine on the other hand. Treatment plan: MPA: $1500 \mathrm{mg} /$ day p.o.. Criteria for pts.' eligibility: a) pts. with favourable prognostic factors (p.f.) before cytotoxic treatment (group 1), b) pts. with unfavourable p.f. failing to cytotoxic treatment (group 2), c) age between $18-75$ years, d) no signs of congestive heart failure or thrombotic diathesis.

From II/82 - XI/84 92 pts. entered the study, 72 are evaluable for response (UICC-criteria), 78 for toxicity (WHOscale). Therapeutic results: group 1: objective remission was observed in 10 of the 23 pts.: complete remission (CR): 4 pts. and partial remission (PR): 6 pts.. Remission duration: median $(\mathrm{m})=60$ weeks $(\mathrm{W})$, stable disease (SD) occured in 9 pts.; 4 pts. experienced progressive disease (PD). Group 2: objective remission was observed in 8 of 49 pts., thereof $1 \mathrm{CR}$. SD occured in 22 pts.. 18 pts. experienced PD. Duration of remission: $m=40 \mathrm{w}$. Pts. with PD presented significantly more often with MPA plasma levels $<100 \mathrm{ng} / \mathrm{m} 7$ than $\mathrm{pts}$. with $\mathrm{CR}$, PR or SD. At the time of the relaps MPA-p.1. dropped to lower values $(m=60$ $\mathrm{ng} / \mathrm{ml})$. Cortisot p.l. Were suppressed in pts. responding to $M P A(m=2,9 \mathrm{ng} / \mathrm{d}\})$ and only reduced in pts. with SD or PD $(m=8 \mathrm{ng} / \mathrm{dl})$. Prolactine $\mathrm{p} .1$. had no strong correlation with treatment response.

Priv.Doz.Dr.K.Possinger, Med. Klinik III, Klinikum Großhadern. Marchioninistr. 15, 8000 München 70; Gesel1schaft für Strahlen- und Umweltforschung, Neuherberg. 